\title{
Minimally Invasive Aortic Valve Replacement Following Root Enlargement on too Narrow Annulus to Perform Transcatheter Aortic Valve Implantation
}

\author{
Kosuke Sakamoto, ${ }^{1} \mathrm{MD}$, Toshinori Totsugawa, ${ }^{1} \mathrm{MD}$, Arudo Hiraoka, ${ }^{1} \mathrm{MD}$, Kentaro Tamura, ${ }^{1} \mathrm{MD}$, \\ Hidenori Yoshitaka, ${ }^{1} \mathrm{MD}$ and Taichi Sakaguchi, ${ }^{1} \mathrm{MD}$
}

\begin{abstract}
Summary
An 88-year-old woman was diagnosed with aortic stenosis and an aortic annulus that was too narrow to perform transcatheter aortic valve implantation. Surgery was performed through a 7-cm right mini-thoracotomy at the fourth intercostal space. A 19-mm aortic valve bioprosthesis was implanted after root enlargement. The fourth intercostal space was a suitable site for aortic root enlargement because of the shorter skin-to-root distance and the detailed exposure of the aortic valve after cutting the aortic wall. This study concluded that minimally-invasive aortic valve replacement following root enlargement can be an option for the treatment of elderly patients with aortic stenosis accompanied by an annulus that is too small to perform transcatheter aortic valve implantation.
\end{abstract}

(Int Heart J 2018; 59: 634-636)

Key words: Aortic stenosis, Small annulus, Minimally invasive cardiac surgery, Intercostal thoracotomy, Patch augmentation

$\mathrm{T}$ echnological advancement and establishment of preoperative evaluation using computed tomography (CT) imaging makes transcatheter aortic valve implantation (TAVI) a safe option for the treatment of aortic stenosis. TAVI is now a widespread surgery for elderly or high-risk patients even in Japan. Furthermore, some clinical trials which have focused on elderly patients at intermediate risk have indicated that the outcome of TAVI is as good as that of conventional surgery. ${ }^{1)}$ However, for anatomical reasons, there exist some cases for which TAVI is inappropriate. We encountered an elderly patient with aortic stenosis in whom TAVI seemed to be inappropriate because of a relatively small aortic annulus. Herein, we present the use of minimally invasive aortic valve replacement (MIAVR) through a right mini-thoracotomy, following root enlargement.

\section{Case Report}

An 88-year-old woman who presented with repeated syncope was diagnosed with severe aortic stenosis and was admitted to our hospital in October 2016. Her blood pressure was $110 / 73 \mathrm{~mm} \mathrm{Hg}$ and her heart rate was 72 beats/minute. A systolic murmur, Levine grade 5/6, was heard at the third intercostal space along the right sternal border. Her Canadian Study of Health and Aging (CSHA) frailty scale score was 5 out of 9 . An electrocardiogram showed normal sinus rhythm with a strain pattern of the
QRS wave. An echocardiography revealed a left ventricular ejection fraction of $78 \%$, a peak aortic valve pressure gradient (AVPG) of $159 \mathrm{~mm} \mathrm{Hg}$, a mean AVPG of 95 $\mathrm{mm} \mathrm{Hg}$, an aortic valve area of $0.49 \mathrm{~cm}^{2}$, and moderate aortic regurgitation. A cardiac $\mathrm{CT}$ scan showed that the aortic annular size was $17.7 \mathrm{~mm}$ in area and $17.9 \mathrm{~mm}$ in circumference (Figure 1A). These measurements were outside of the range covered by the Edwards Sapien 3 or Medtronic CoreValve, the transcatheter heart valves available at that time in Japan. Our cardiac team recommended surgical aortic valve replacement with root enlargement. A contrast-enhanced CT scan showed rare atherosclerotic changes in the aorta; therefore, considering the physical activity of the patient, we decided to perform the surgery through a right mini-thoracotomy.

Although MIAVR is usually performed through the third intercostal space, our surgical procedure utilized a 7 $\mathrm{cm}$, right anterolateral thoracotomy at the fourth intercostal space instead (Figure 1B). Cardiopulmonary bypass was established via femoro-femoral bypass, and the ascending aorta was clamped with a flexible clamp. Cardiac arrest was achieved by antegrade administration of crystalloid cardioplegic solution. After an oblique aortotomy, the aortic wall was widely opened using three 5-0 polypropylene pledgeted traction sutures; then the aortic valve was well exposed (Figure 2A). The calcified cusps were resected using long scissors, and the annular calcifications were completely removed by an ultrasonic aspirator. How-

From the ${ }^{1}$ Department of Cardiovascular Surgery, The Sakakibara Heart Institute of Okayama, Okayama, Japan.

Address for correspondence: Toshinori Totsugawa, MD, Department of Cardiovascular Surgery, The Sakakibara Heart Institute of Okayama, 2-5-1 Nakaicho, Kita-ku, Okayama, 700-0804, Japan. E-mail: toshinoritotsugawa@gmail.com

Received for publication July 4, 2017. Revised and accepted August 25, 2017.

Released in advance online on J-STAGE May 9, 2018.

doi: 10.1536/ihj.17-371

All rights reserved by the International Heart Journal Association. 

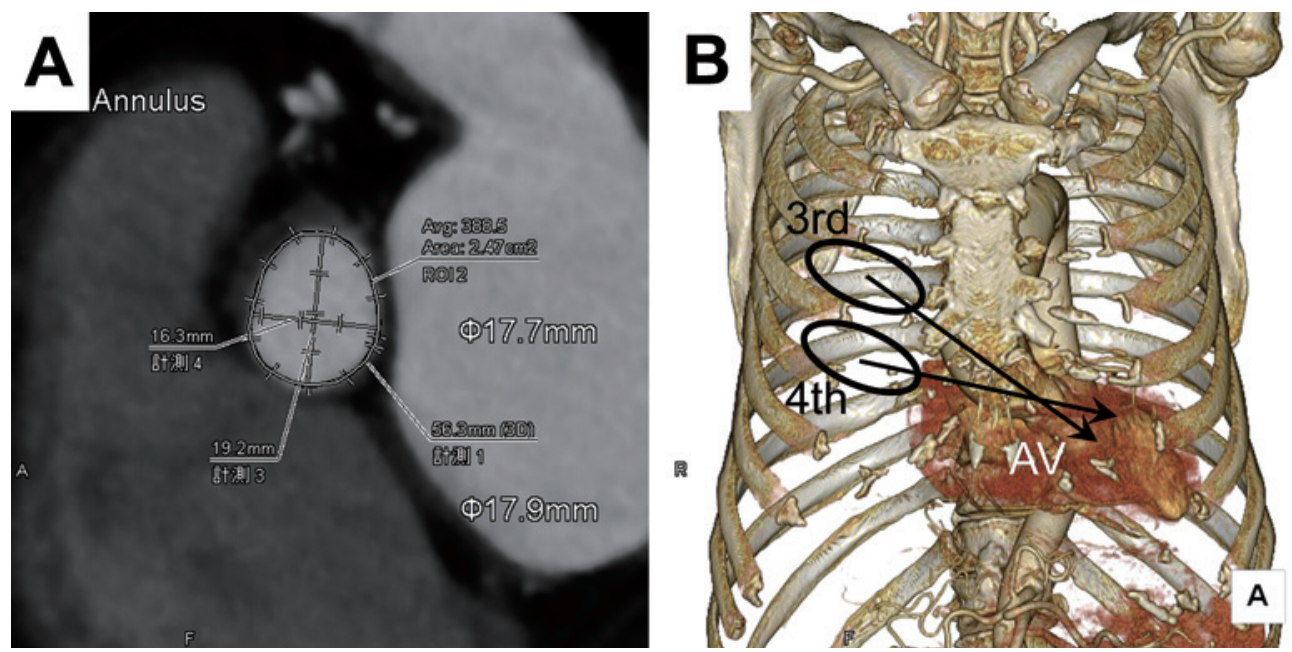

Figure 1. A: Annular measurement by cardiac computed tomography scan. The aortic annular size was $17.7 \mathrm{~mm}$ in area and $17.9 \mathrm{~mm}$ in circumference. B: Three-dimensional CT scan showing the relationship between the thoracotomy and the aortic root, and the difference between the thoracotomies. MIAVR is usually performed from the third intercostal space, so that the aortic valve, the aortotomy and the thoracotomy are located along a straight line. However, in the present case, surgery was performed through a right anterolateral thoracotomy at the fourth intercostal space in consideration of the access to the aortic root. AV indicates aortic valve.
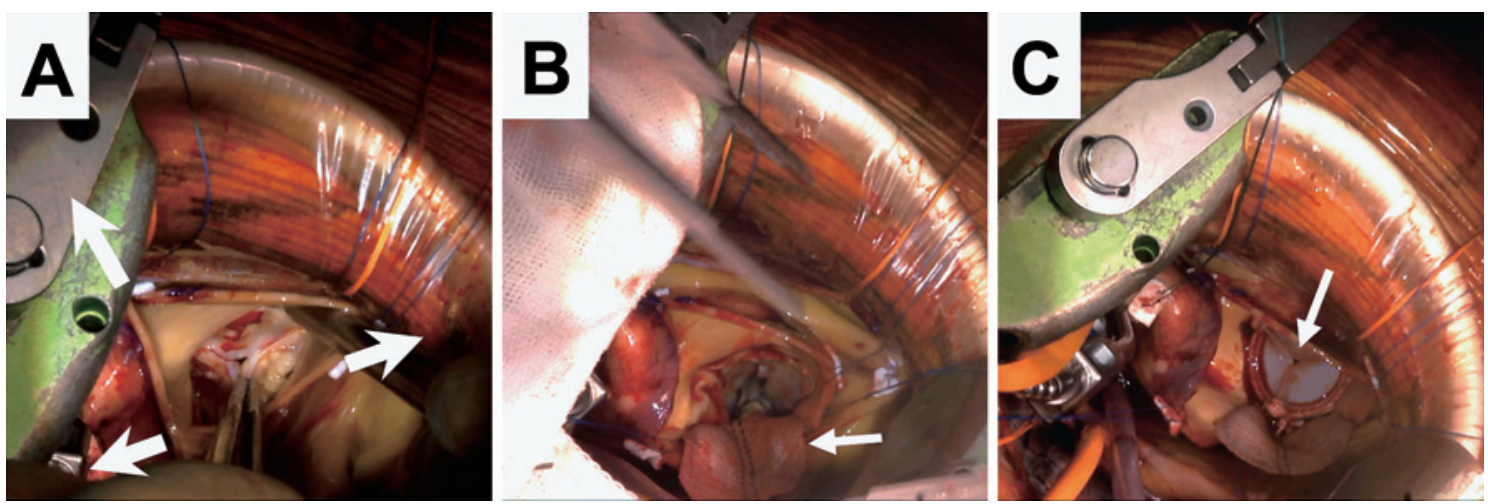

Figure 2. A: After an oblique aortotomy, the aortic wall was retracted in three directions (arrows); after this, the aortic valve was well-exposed B: A teardrop-shaped Dacron patch (arrow) was sutured along the aortic wall. C: Finally, a 19-mm bioprosthesis (arrow) was implanted in a supra-annular position.

ever, the 19-mm-bioprosthetic sizers, both the CarpentierEdwards PERIMOUNT Magna Ease Aortic Heart Valve and the Medtronic Mosaic Ultra Aortic Heart Valve, were unable to pass through the annulus. Next, the aortic root was cut open at the left coronary cusp-noncoronary cusp commissure and a teardrop-shaped Dacron patch was sutured along the aortic wall (Figure 2B). To prevent potentially lethal bleeding from the edge of the patch, three 4-0 polypropylene mattress sutures with felt pledget reinforcement were placed at the bottom of the aortic root, and the patch was then sutured using a double-layer method combining a horizontal mattress with over-and-over suturing. A 19-mm Carpentier-Edwards PERIMOUNT Magna Ease Aortic Heart Valve (Edwards Lifesciences Co., Irvine, CA, USA) was subsequently implanted in a supra-annular position (Figure 2C). The durations of the operation, cardiopulmonary bypass, and aortic cross-clamping were 304, 209, and 130 minutes, respectively.
Although the patient developed complete atrioventricular block immediately after surgery, she recovered sinus rhythm without pacemaker implantation one week after surgery. She returned to normal activities of daily living within 10 days; however, she strongly preferred rehabilitation at our hospital and was discharged 23 days after surgery. Postoperative echocardiography revealed a left ventricular ejection fraction of $77 \%$, a mean AVPG of 5 $\mathrm{mmHg}$, and no aortic regurgitation.

\section{Discussion}

Recently, TAVI has gained rapid popularity for elderly or high-risk patients, including in Japan. However, as was the case here, an aortic annulus less than $18 \mathrm{~mm}$ is relatively narrow for TAVI. The range of aortic annulus diameters available for TAVI at that time were 18.6-21 $\mathrm{mm}$ for the 20-mm Sapien 3 heart valve, and 20-23 mm 
for the 26-mm CoreValve device; therefore, the sub-18 $\mathrm{mm}$ size of the aortic annulus is below the size range that standard TAVI devices cover. Thus, TAVI was contraindicated due to the risk of annular rupture, ${ }^{3)}$ and we could find no reports regarding an undersized TAVI useful for such a narrow annulus. Surgical aortic valve replacement including root enlargement is the last option for such cases. Although our patient was an elderly and slightly frail woman with a CSHA clinical frailty scale score of 5, a preoperative CT scan showed rare atherosclerotic changes in the aorta. Gilmanov, et al. reported good results of MIAVR performed through a right anterior thoracotomy compared with a median sternotomy, even in octogenarians. ${ }^{4}$ In our hospital, small annulus requiring root enlargement has traditionally been a contraindication of MIAVR. ${ }^{2)}$ Kitamura, et al. reported that moderate prosthesis-patient mismatch did not have negative impact on survival or reduction of left ventricular mass index in elderly patients, ${ }^{5)}$ however, aortic valve replacement using a small mechanical valve would deteriorate quality of life in elderly patients. Based on our experience of performing more than 200 cases of MIAVR, we decided to perform aortic valve replacement with a bioprosthesis following root enlargement through a right mini-thoracotomy for the patient. The mean aortic cross-clamping time in MIAVR via an anterolateral thoracotomy is approximately 100 minutes; ${ }^{2)}$ aortic root enlargement takes 30 minutes extra. The main drawback of this minimally invasive surgery is the longer cross-clamping time, so the use of MIAVR for elderly patients is controversial. However, avoiding sternotomy-related complications such as sternal dehiscence is especially useful in elderly women with osteoporosis, and can contribute to their early rehabilitation.

The third intercostal space is now the most popular intercostal space for MIAVR via anterolateral thoracotomy in our institute. In this approach, the aortic valve, the aortotomy, and the thoracotomy are located along a straight line. ${ }^{6}$ However, in the present case, the surgery was performed through a mini-thoracotomy at the fourth intercostal space. To prevent hemorrhagic complications, we regard adequate access to the aortic root as the most important factor in the successful performance of this procedure. Entry through the fourth intercostal space is usually unfavorable to achieving adequate exposure of the aortic valve, as the aortic wall interferes with visualizing the valve. On the contrary, the space is suitable for use in root enlargement due to the shorter skin-to-root distance. Exposure of the aortic valve was also improved after cutting the aortic wall and applying three traction sutures. Bleeding from the edge of the cut aortic root is a lethal complication in this procedure; therefore, careful maneuvering with the use of felt pledget reinforcement and doublelayer suturing is mandatory. Maximizing exposure was helpful for the fine suturing of the Dacron patch and the placement of annular stitches in the present case.

\section{Conclusion}

We performed MIAVR with root enlargement safely on a slightly frail elderly patient, and her postoperative recovery was uneventful. We therefore found that MIAVR following root enlargement can be an option for the treatment of elderly patients with aortic stenosis combined with a narrow annulus that precludes TAVI.

\section{Disclosures}

Conflicts of interest: The authors declare that they have no conflict of interest.

\section{References}

1. Leon MB, Smith CR, Mack MJ, et al. Transcatheter or surgical aortic-valve replacement in intermediate-risk patients. N Engl J Med 2016; 374: 1609-20.

2. Totsugawa T, Kuinose M, Hiraoka A, Yoshitaka H, Tamura K, Sakaguchi T. Anterolateral approach for minimally invasive aortic valve replacement. Gen Thorac Cardiovasc Surg 2014; 62: 290-5.

3. Marco B, Tae-Hyun Y, Josep RC, et al. Anatomical and procedural features associated with aortic root rupture during balloonexpandable transcatheter aortic valve replacement. Circulation 2013; 128: 244-53.

4. Gilmanov D, Farneti PA, Ferrarini M, et al. Full sternotomy versus right anterior minithoracotomy for isolated aortic valve replacement in octogenarians: a propensity-matched study. Interact Cardiovasc Thorac Surg 2015; 20: 732-41.

5. Kitamura $\mathrm{T}$, Torii $\mathrm{S}$, Hanayama $\mathrm{N}$, et al. Moderate prosthesispatient mismatch may be negligible in elderly patients undergoing conventional aortic valve replacement for aortic stenosis. Int Heart J 2013; 54: 11-4.

6. Totsugawa T, Suzuki K, Hiraoka A, Tamura K, Yoshitaka H, Sakaguchi T. Concomitant septal myectomy during minimally invasive aortic valve replacement through a right minithoracotomy for the treatment of aortic stenosis with systolic anterior motion of the mitral valve. Gen Thorac Cardiovasc Surg 2017; 65: 657-60. 